\title{
Better Vision Through Manipulation
}

\author{
Giorgio Metta*,** \\ *LIRA-Lab, DIST \\ University of Genova \\ Viale F. Causa, 13 \\ 16145 Genova, Italy
}

\author{
Paul Fitzpatrick** \\ ${ }^{* *}$ MIT AI Lab \\ 200 Technology Square \\ Cambridge, MA 02139 US
}

\begin{abstract}
For the purposes of manipulation, we would like to know what parts of the environment are physically coherent ensembles - that is, which parts will move together, and which are more or less independent. It takes a great deal of experience before this judgement can be made from purely visual information. This paper develops active strategies for acquiring that experience through experimental manipulation, using tight correlations between arm motion and optic flow to detect both the arm itself and the boundaries of objects with which it comes into contact. We argue that following causal chains of events out from the robot's body into the environment allows for a very natural developmental progression of visual competence, and relate this idea to results in neuroscience.
\end{abstract}

\section{Introduction}

A robot is an actor in its environment and not simply a passive observer. This gives it the potential to examine the world using causality, by performing probing actions and learning from the response. Tracing chains of causality from motor action to perception (and back again) is important both to understand how the brain deals with sensorimotor coordination and to implement those same functions in an artificial system, such as a humanoid robot.

In this paper, we propose that such causal probing can be arranged in a developmental sequence leading to a manipulation-driven representation of objects. We present results for two important steps along the way, and describe how we plan to proceed.

Table 1 shows three levels of causal complexity. The simplest causal chain that the robot experiences is the perception of its own actions. The temporal aspect is immediate: visual information is tightly synchronized to motor commands. We use this strong correlation to identify parts of the robot body specifically, the end-point of the arm.

Once this causal connection is established, we can go further and use it to active explore the boundaries of objects. In this case, there is one more step in the causal chain, and the temporal nature of the response may be delayed since initiating a reaching movement doesn't immediately elicit consequences in the environment.

Finally we argue that extending this causal chain further will allow us to approach the representational power of "mirror neurons" (Fadiga et al., 2000), where a connection is made between our own actions and the actions of another.

\section{The elusive object}

Sensory information is intrinsically ambiguous, and very distant from the world of well-defined objects in which humans believe they live. What criterion should be applied to distinguish one object from another? How can perception support such a phenomenon as figure-ground segmentation? Consider the example in Figure 1. It is immediately clear that the drawing on the left is a cross, perhaps because we already have a criterion, which allows segmenting on the basis of the intensity difference. It is slightly less clear that the zeros and ones on the middle panel are still a cross. What can we say about the array on the right? If we are not told, and we do not have the criterion to perform the figure-ground segmentation, we might think this is just a random collection of numbers. But if we are told that the criterion is "prime numbers vs. non-prime" then a cross can still be identified.

While we have to be inventive to come up with a segmentation problem that tests a human, we don't have to go far at all to find something that baffles our robots. Figure 2 shows a robot's-eye view of a cube sitting on a table. Simple enough, but many rules of thumb used in segmentation fail in this particular case. And even an experienced human observer, diagnosing the cube as a separate object based on its shadow and subtle differences in the surface texture of the cube and table, could in fact be mistaken perhaps some malicious researcher is up to mischief. The only way to find out for sure is to take action, and start poking and prodding. As early as 1734, Berkeley observed that:

...objects can only be known by touch. Vision 


\begin{tabular}{|l|l|l|}
\hline type & nature of causation & time profile \\
\hline \hline sensorimotor coordination & direct causal chain & strict synchrony \\
\hline object probing & one level of indirection & $\begin{array}{l}\text { fast onset upon contact, poten- } \\
\text { tial for delayed effects }\end{array}$ \\
\hline mirror representation & $\begin{array}{l}\text { complex causation involving } \\
\text { multiple causal chains }\end{array}$ & $\begin{array}{l}\text { arbitrarily delayed onset and ef- } \\
\text { fects }\end{array}$ \\
\hline
\end{tabular}

Table 1: Degrees of causal indirection. There is a natural trend from simpler to more complicated tasks. The more time-delayed an effect, the more difficult it is to model.

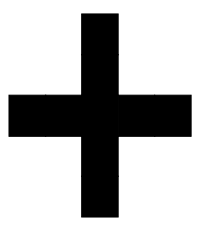

a cross

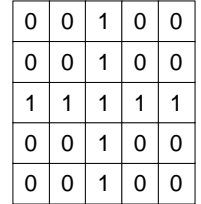

a binary cross

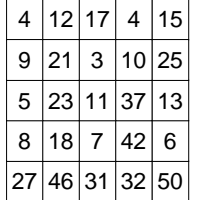

$?$
Figure 1: Three examples of crosses, following (Manzotti and Tagliasco, 2001). The human ability to segment objects is not general-purpose, and improves with experience.

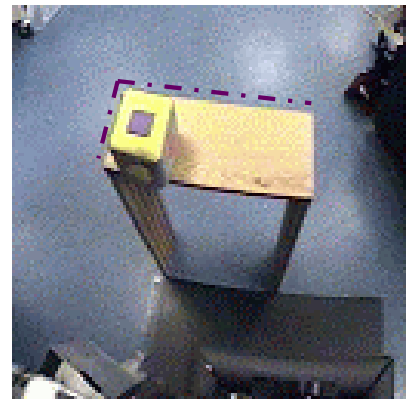

Figure 2: A cube on a table. The edges of the table and cube happen to be aligned (dashed line), the colors of the cube and table are not well separated, and the cube has a potentially confusing surface pattern.

is subject to illusions, which arise from the distance-size problem... (Berkeley, 1972)

In this paper, we provide support for a more nuanced proposition: that in the presence of touch, vision becomes more powerful, and many of its illusions fade away.

\section{Objects and actions}

The example of the cross composed of prime numbers is a novel (albeit unlikely) type of segmentation in our experience as adult humans. We might imagine that when we were very young, we had to initially form a set of such criteria to solve the object identification/segmentation problem in more mundane circumstances. That such abilities develop and are not completely innate is suggested by results in neural science. For example Kovacs (Kovacs, 2000) has shown that perceptual grouping is slow to develop and continues to improve well beyond early childhood (14 years). Long-range contour integration was tested and this work elucidated how this ability develops to enable extended spatial grouping.

Key to understanding how such capabilities could develop is the well-known result by Ungerleider and Mishkin (Ungerleider and Mishkin, 1982) who first formulated the hypothesis that objects are represented differently during action than they are for a purely perceptual task. Briefly, they argue that the brain's visual pathways split into two main streams: the dorsal and the ventral (Milner and Goodale, 1995). The dorsal deals with the information required for action, while the ventral is important for more cognitive tasks such as maintaining an object's identity and constancy. Although the dorsal/ventral segregation is emphasized by many commentators, it is significant that there is a great deal of cross talk between the streams. Observation of agnosic patients (Jeannerod, 1997) shows a much more complicated relationship than the simple dorsal/ventral dichotomy would suggest. For example, although some patients could not grasp generic objects (e.g. cylinders), they could correctly preshape the hand to grasp known objects (e.g. a lipstick): interpreted in terms of the two pathways, this implies that the ventral representation of the object can supply the dorsal stream with size information.

The dorsal stream goes through the parietal lobe and premotor cortex, which project heavily onto the primary motor cortex to eventually control movements. For many years the premotor cortex was considered just another big motor area. Recent studies (Jeannerod, 1997) have demonstrated that this is not the case. Visually responsive neurons have been found: some are purely visual, but many have significant visuo-motor characteristics. In area F5 in the monkey, neurons responding to object manipulation gestures are found. They can be classified in at least two different types: canonical and mirror. The canonical type is active in two situations: i) when grasping an object and ii) when fixating that same object. For example, a neuron active when grasping a ring also fires when the monkey simply looks at the 
ring. This could be thought of as a neural analogue of the "affordances" of Gibson (Gibson, 1977).

The second type of neuron, the mirror neuron (Fadiga et al., 2000), becomes active under two conditions: i) when manipulating an object (e.g. grasping it), and ii) when watching someone else performing the same action on the same object. This is a more subtle representation of objects, which allows and supports, at least in theory, mimicry behaviors. In human, area F5 is thought to correspond to Broca's area: there is an intriguing link between gesture understanding, language, imitation, and mirror neurons (Rizzolatti and Arbib, 1998).

Another important class of neurons in premotor cortex is found in area F4 (Fogassi et al., 1996). While F5 is more concerned with the distal muscles (i.e. the hand), F4 controls more proximal muscles (i.e. reaching). A subset of neurons in F4 has a somatosensory, visual, and motor receptive field. The visual receptive field (RF) extends in $3 \mathrm{D}$ from a given body part, for example, the forearm. The somatosensory RF is usually in register with the visual one. Finally, motor information is integrated into the representation by maintaining the receptive field anchored to the correspondent body part (the forearm in this example) irrespective of the relative position of the head and arm.

\section{A working hypothesis}

Taken together this results from neuroscience suggest a very basic role for motor action. Certainly vision and action are intertwined at a very basic level. While an experienced adult can interpret visual scenes perfectly well without acting upon them, linking action and perception seems crucial to the developmental process that leads to that competence. We can construct a working hypothesis: that action is required to object recognition in cases where an agent has to develop categorization autonomously. Of course in standard supervised learning action is not required since the trainer does the job of presegmenting the data by hand. In an ecological context, some other mechanism has to be provided. Ultimately this mechanism is the body itself that through action (under some suitable developmental rule) generates informative percepts.

Neurons in area F4 are thought to provide a body map useful for generating arm, head, and trunk movements. Our robot learns autonomously a crude version of this body map by fusing vision and proprioception. As a step towards establishing the kind of visuomotor representations seen in F5, we then develop a mechanism for using reaching actions to visually probe the connectivity and physical extent of objects without any prior knowledge of the appearance of the objects (or indeed of the arm itself).

\section{The experimental platform}

This work is implemented on the robot Cog, an upper torso humanoid (Brooks et al., 1999). The robot has previously been applied to tasks such as visually-guided pointing (Marjanović et al., 1996), and rhythmic operations such as turning a crank or driving a slinky (Williamson, 1998). Cog has two arms, each of which has six degrees of freedom two per shoulder, elbow, and wrist. The joints are driven by series elastic actuators (Williamson, 1995) - essentially a motor connected to its load via a spring (think strong and torsional rather than loosely coiled). The arm is not designed to enact trajectories with high fidelity. For that a very stiff arm is preferable. Rather, it is designed to perform well when interacting with a poorly characterized environment, where collisions are frequent and informative events.
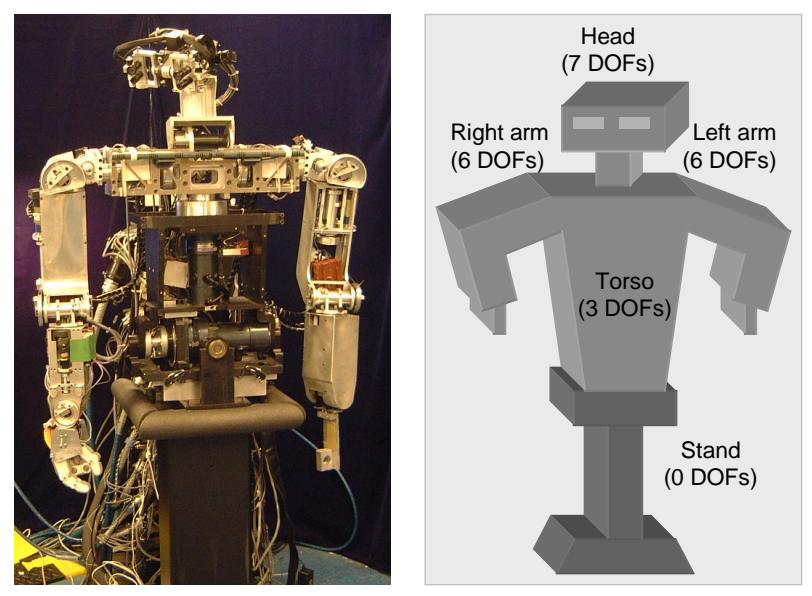

Figure 3: Degrees of freedom (DOFs) of the robot Cog. The arms terminate either in a primitive "flipper" or a four-fingered hand. The head, torso, and arms together contain 22 degrees of freedom.

\section{Perceiving direct effects of action}

Motion of the arm may generate optic flow directly through the changing projection of the arm itself, or indirectly through an object that the arm is in contact with. While the relationship between the optic flow and the physical motion is likely to be extremely complex, the correlation in time of the two events will generally be exceedingly precise. This time-correlation can be used as a "signature" to identify parts of the scene that are being influenced by the robot's motion, even in the presence of other distracting motion sources. In this section, we show how this tight correlation can be used to localize the arm in the image without any prior information about visual appearance. In the next section we will show that once the arm has been localized we can go further, and identify the boundaries of objects with which the arm comes into contact. 


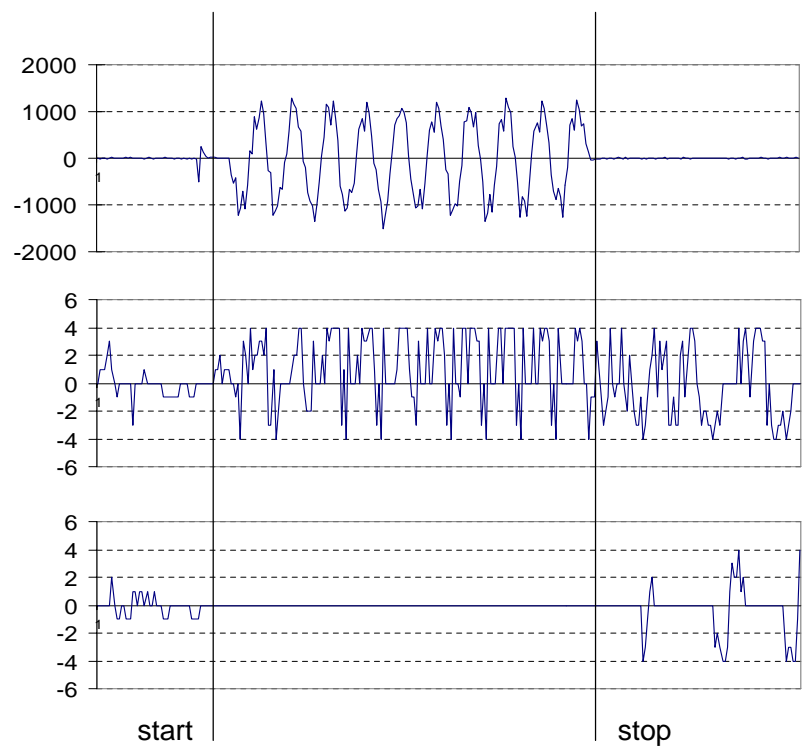

Figure 4: An example of the correlation between optic flow and arm movement. The traces show the movement of the wrist joint (upper plot) and optic flow sampled on the arm (middle plot) and away from it (lower plot). As the arm generates a repetitive movement, the oscillation is clearly visible in the middle plot and absent in the lower. Before and after the movement the head is free to saccade, generating the other spikes seen in the optic flow.

\section{Reaching out}

The first step towards manipulation is to reach objects within the workspace. If we assume targets are chosen visually, then ideally we need to also locate the end-effector visually to generate an error signal for closed-loop control. Some element of open-loop control is necessary since the end-point may not always be in the field of view (for example, when it is in its the resting position), and the overall reaching operation can be made faster with a feed-forward contribution to the control.

The simplest possible open loop control would map directly from a fixation point to the arm motor commands needed to reach that point (Metta et al., 1999) using a stereotyped trajectory, perhaps using postural primitives (Mussa-Ivaldi and Giszter, 1992). If we can fixate the end-effector, then it is possible to to learn this map by exploring different combinations of direction of gaze vs. arm position (Marjanović et al., 1996, Metta et al., 1999). So locating the end-effector visually is key both to closed-loop control, and to training up a feedforward path. We shall demonstrate that this localization can be performed without knowledge of the arm's appearance, and without assuming that the arm is the only moving object in the scene.

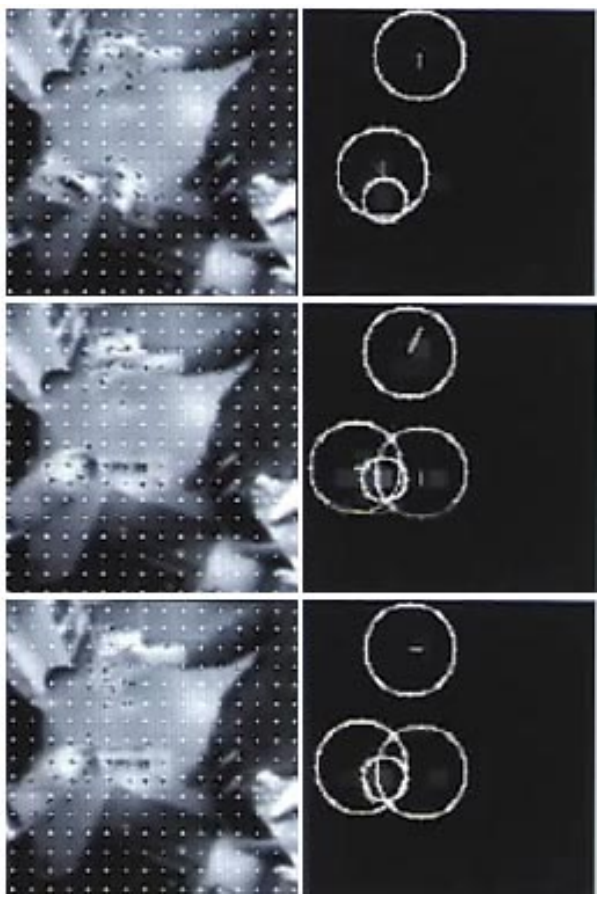

Figure 5: Detecting the arm/gripper through motion correlation. The robot's point of view and the optic flow generated are shown on the left. On the right are the results of correlation. Large circles represent the results of applying a region growing procedure to the optic flow. Here the flow corresponds to the robot's arm and the experimenter's hand in the background. The small circle marks the point of maximum correlation, identifying the regions that correspond to the robot's own arm.

\section{Localizing the arm visually}

The robot is not a passive observer of its arm, but rather the initiator of its movement. This can be used to distinguish the arm from parts of the environment that are more weakly affected by the robot. The arm of a robot was detected in (Marjanović et al., 1996) by simply waving it and assuming it was the only moving object in the scene. We take a similar approach here, but use a more stringent test of looking for optic flow that is correlated with the motor commands to the arm. This allows unrelated movement to be ignored. Even if a capricious engineer where to replace the robot's arm with one of a very different appearance, and then stand around waving the old arm, this detection method will not be fooled.

The actual relationship between arm movements and the optic flow they generate is complex. Since the robot is in control of the arm, it can choose to move it in a way that bypasses this complexity. In particular, if the arm rapidly reverses direction, the optic flow at that instant will change in sign, giving a tight, clean temporal correlation. Since our op- 


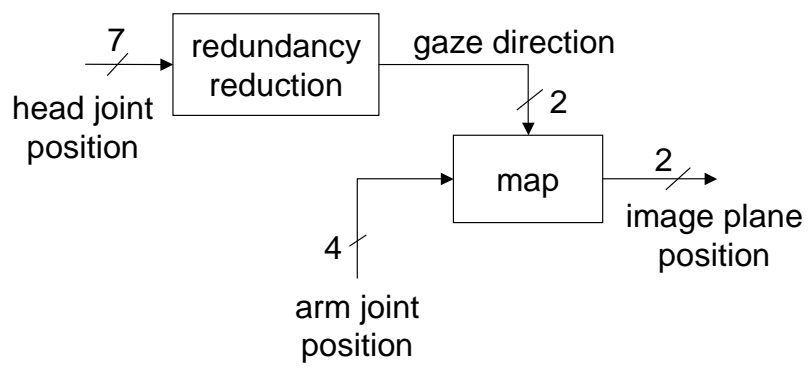

Figure 6: Mapping from proprioceptive input to a visual prediction. Head and arm joint positions are used to estimate the position of the projection of the hand in the image plane. Redundant configurations of the (7 DOF) head are mapped to a simpler (2D) representation, and the wrist-related DOFs of the arm are ignored.

tic flow processing is coarse (a $16 \times 16$ grid over a $128 \times 128$ image at $15 \mathrm{~Hz}$ ), we simply repeat this reversal a number of times to get a strong correlation signal during training. With each reversal the probability of correlating with unrelated motion in the environment goes down. This probability could also be reduced by higher resolution (particularly in time) visual processing.

Figure 4 shows an example of this procedure in operation, comparing the velocity of the arm's wrist with the optic flow at two positions in the image plane. A trace taken from a position away from the arm shows no correlation, while conversely the flow at a position on the wrist is strongly different from zero over the same period of time. Figure 5 shows examples of detection of the arm and rejection of a distractor.

\section{Localizing the arm using proprioception}

The localization method for the arm described so far relies on a relatively long "signature" movement that would slow down reaching. This can be overcome by training up a function to estimate the location of the arm in the image plane from proprioceptive information (joint angles) during an exploratory phase, and using that to constrain arm localization during actual operation. As a function approximator we simply fill a look-up table, reducing the 11dimensional input space of joint angles based on the much lower number of degrees of freedom used in controlling them (see Figure 6). Figure 7 shows the resulting behavior after about twenty minutes of realtime learning.

\section{Perceiving indirect effects of action}

We have assumed that the target of a reaching operation is chosen visually. As discussed in the introduction, visual segmentation is not easy, so we should

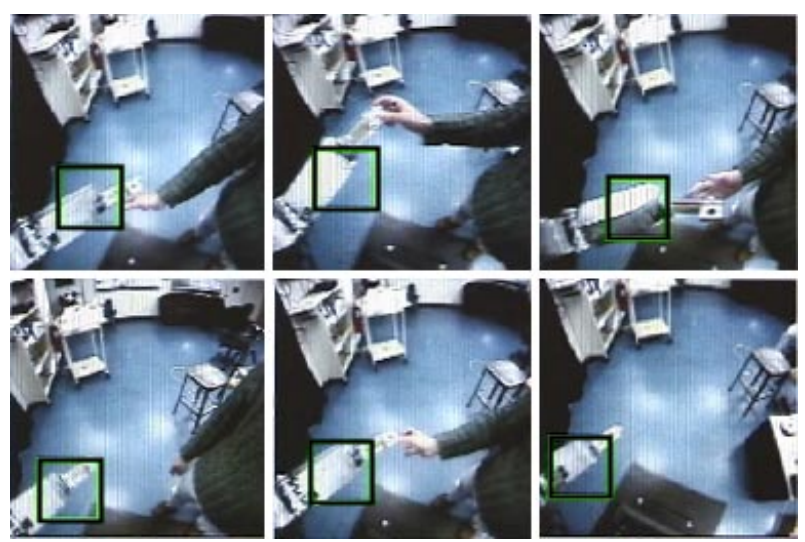

Figure 7: Predicting the location of the arm in the image as the head and arm change position. The rectangle represents the predicted position of the arm using the map learned during a twenty-minute training run. The predicted position just needs to be sufficiently accurate to initialize a visual search for the exact position of the end-effector.

not expect a target selected in this way to be a correctly segmented. For the example scene in Figure 2 (a cube sitting on a table), the small inner square on the cube's surface pattern might be selected as a target. The robot can certainly reach towards this target, but grasping it would prove difficult without a correct estimate of the object's physical extent. In this section, we develop a procedure for refining the segmentation using the same idea of correlated motion used earlier to detect the arm.

When the arm enters into contact with an object, one of several outcomes are possible. If the object is large, heavy, or otherwise unyielding, motion of the arm may simply be resisted without any visible effect. Such objects can simply be ignored, since the robot will not be able to manipulate them. But if the object is smaller, it is likely to move a little in response to the nudge of the arm. This movement will be temporally correlated with the time of impact, and will be connected spatially to the endeffector - constraints that are not available in passive scenarios (Birchfield, 1999). If the object is reasonably rigid, and the movement has some component in parallel to the image plane, the result is likely to be a flow field whose extent coincides with the physical boundaries of the object.

Figure 8 shows how a "poking" movement can be used to refine a target. During a poke operation, the arm begins by extending outwards from the resting position. The end-effector (or "flipper") is localized as the arm sweeps rapidly outwards, using the heuristic that it lies at the highest point of the region of optic flow swept out by the arm in the image (the head orientation and reaching trajectory are controlled so that this is true). The arm is driven 


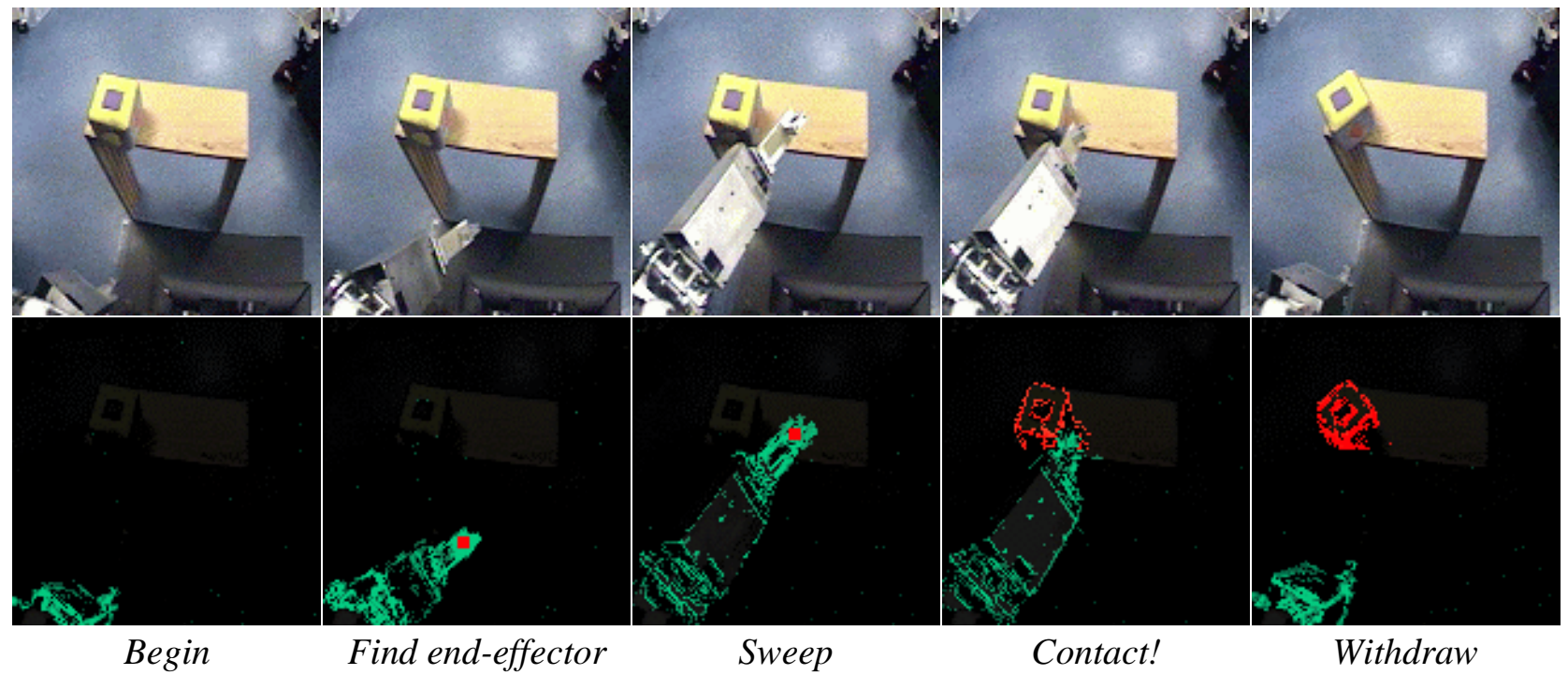

Figure 8: The upper sequence shows an arm extending into a workspace, tapping an object, and retracting. This is an exploratory mechanism for finding the boundaries of objects, and essentially requires the arm to collide with objects under normal operation, rather than as an occasional accident. The lower sequence shows the shape identified from the tap using simple image differencing and flipper tracking.

outward into the neighborhood of the target which we wish to define, stopping if an unexpected obstruction is reached. If no obstruction is met, the flipper makes a gentle sweep of the area around the target. This minimizes the opportunity for the motion of the arm itself to cause confusion; the motion of the flipper is bounded around the endpoint whose location we know from tracking during the extension phase, and can be subtracted easily. Flow not connected to the end-effector can be ignored as a distractor.

For simplicity, the head is kept steady throughout the poking operation, so that simple image differencing can be used to detect motion at a higher resolution than optic flow. Because a poking operation currently always starts from the same location, the arm is localized using a simple heuristic rather than the procedure described in the previous section - the first region of optic flow appearing in the lower part of the robot's view when the reach begins is assumed to be the arm.

The poking operation gives clear results for a rigid object that is free to move. What happens for nonrigid objects and objects that are attached to other objects? Here the results of poking are likely to be more complicated to interpret - but in a sense this is a good sign, since it is in just such cases that the idea of an object becomes less well-defined. Poking has the potential to offer an operational theory of "objecthood" that is more tractable than a vision-only approach might give, and which cleaves better to the true nature of physical assemblages. The idea of a physical object is rarely completely coherent, since it depends on where you draw its boundary and that
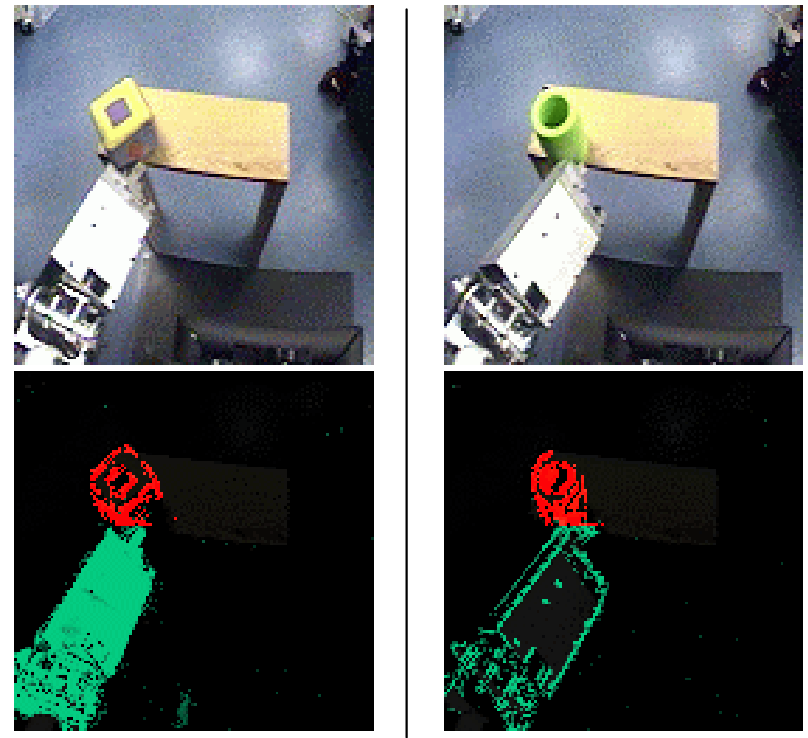

Figure 9: Poking can reveal a diffence in the shape of two objects without any prior knowledge of their appearance.

may well be task-dependent. Poking allows us to determine the boundary around a mass that moves together when disturbed, which is exactly what we need to know for manipulation. As an operational definition of object, this has the attractive property of breaking down into ambiguity in the right circumstances - such as for large interconnected messes, floppy formless ones, liquids, and so on. 


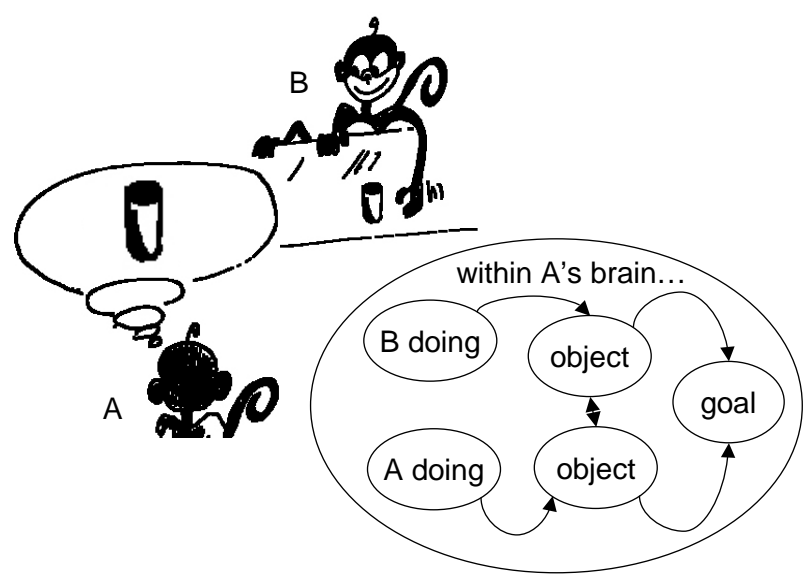

Figure 10: Mirror neurons and causality: from the observer's point of view (A), understanding B's action means mapping it onto the observer's own motor repertoire. If the causal chain leading to the goal is already in place (lower branch of the graph) then the acquisition of a mirror neuron for this particular action/object is a matter of building and linking the upper part of the chain to the lower one. There are various opportunities to reinforce this link either at the object level, at the goal level or both.

\section{Developing mirror neurons?}

Poking moves us one step outwards on a causal chain away from the robot and into the world, and gives a simple experimental procedure for segmenting objects. There are many possible elaborations of this method (some are mentioned in the conclusions), all of which lead to a vision system that is tuned to acquiring data about an object by seeing it manipulated by the robot. An interesting question then is whether the system could extract useful information from seeing an object manipulated by someone else. In the case of poking, the robot needs to be able to estimate the moment of contact and to track the arm sufficiently well to distinguish it from the object being poked. We are interested in how the robot might learn to do this. One approach is to chain outwards from an object the robot has poked. If someone else moves the object, we can reverse the logic used in poking - where the motion of the manipulator identified the object - and identify a foreign manipulator through its effect on the object. Poking is an ideal testbed for future work on this, since it is much simpler than full-blown object manipulation and would only require a very simple model of the foreign manipulator to work.

There is considerable precedent in the literature for a strong connection between viewing object manipulation performed by either oneself or another (Wohlscläger and Bekkering, 2002). As we already mentioned F5 contains a class of neurons called canonical neurons that have a very specific response when an object is being either manipulated or fixated. Grossly simplifying, we might think of canonical neurons as an association table of grasp/manipulation (action) types with object (vision) types. Another class of neurons called "mirror neurons" can then be thought of as a second-level association map which links together the observation of a manipulative action performed by somebody else with the neural representation of one's own action.

Figure 10 shows this causal chain in action. There are a series of interesting behaviors that can be realized based on mirror neurons. Mimicry is an obvious application, since it requires just this type of mapping between other and self in terms of motor actions. Another important application is the prediction of future behavior from current actions, or even inverting the causal relation to find the action that most likely will get to the desired consequence.

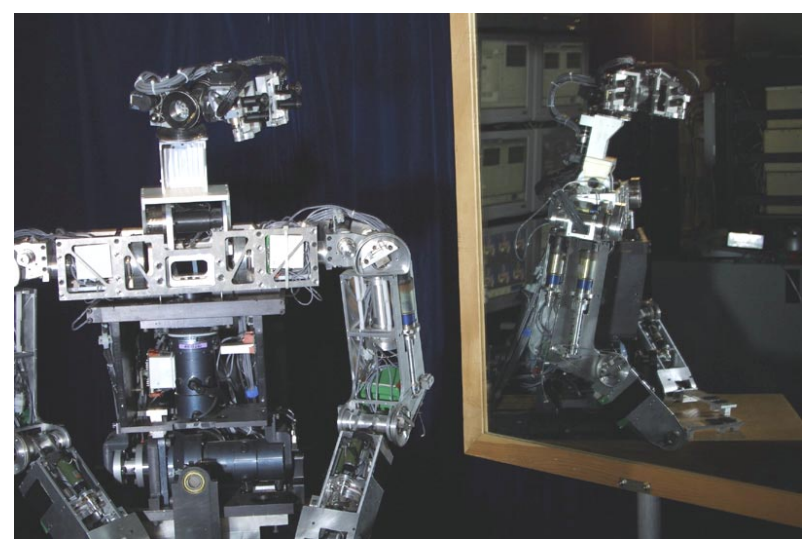

Figure 11: The ultimate goal of this work is for our robot to follow chains of causation outwards from its own simple body into the complex world.

\section{Discussion and Conclusions}

In this paper, we showed how causality can be probed at different levels by the robot. Initially the environment was the body of the robot itself, then later a carefully circumscribed interaction with the outside world. This is reminiscent of Piaget's distinction between primary and secondary circular reactions (Ginsburg and Opper, 1978). Objects are central to interacting with the ouside world. We raised the issue of how an agent can autonomously acquire a working definition of objects.

In computer vision there is much to be gained by bringing a manipulator into the equation. Many variants and extensions to the experimental "poking" strategy explored here are possible. For example, a robot might try to move an arm around behind the object. As the arm moves behind the object, it reveals its occluding boundary. This is a precursor to 
visually extracting shape information while actually manipulating an object, which is more complex since the object is also being moved and partially occluded by the manipulator. Another possible strategy that could be adopted as a last resort for a confusing object might be to simply hit it firmly, in the hopes of moving it some distance and potentially overcoming local, accidental visual ambiguity. Obviously this strategy cannot always be used! But there is plenty of room to be creative here.

\section{Acknowledgements}

This work benefited from discussions with Charles Kemp and Giulio Sandini. Many people have contributed to developing the $\mathrm{Cog}$ platform (Brooks et al., 1999). Funds for this project were provided by DARPA as part of the "Natural Tasking of Robots Based on Human Interaction Cues" project under contract number DABT 63-00-C10102, and by the Nippon Telegraph and Telephone Corporation as part of the NTT/MIT Collaboration Agreement.

\section{References}

Berkeley, G. (1972). A new theory of vision and other writings. Dent, London. First published in 1734 .

Birchfield, S. (1999). Depth and Motion Discontinuities. PhD thesis, Dept. of Electrical Engineering, Stanford University.

Brooks, R. A., Breazeal, C., Marjanovic, M., and Scassellati, B. (1999). The Cog project: Building a humanoid robot. Lecture Notes in Computer Science, 1562:52-87.

Fadiga, L., Fogassi, L., Gallese, V., and Rizzolatti, G. (2000). Visuomotor neurons: ambiguity of the discharge of 'motor' perception? International Journal of Psychophysiology, 35:165-177.

Fogassi, L., Gallese, V., Fadiga, L., Luppino, G., Matelli, M., and Rizzolatti, G. (1996). Coding of peripersonal space in inferior premotor cortex (area F4). Journal of Neurophysiology, pages 141-157.

Gibson, J. J. (1977). The theory of affordances. In Shaw, R. and Bransford, J., (Eds.), Perceiving, acting and knowing: toward an ecological psychology, pages 67-82. Hillsdale NJ: Lawrence Erlbaum Associates Publishers.

Ginsburg, H. and Opper, S. (1978). Piaget's theory of intellectual development. Prentice-Hall, Englewood Cliffs, NJ. 2nd edition.
Jeannerod, M. (1997). The Cognitive Neuroscience of Action. Blackwell Publishers Inc., Cambridge Massachusetts and Oxford UK.

Kovacs, I. (2000). Human development of perceptual organization. Vision Research, 40(1012):1301-1310.

Manzotti, R. and Tagliasco, V. (2001). Coscienza e realtà: una teoria della coscienza per costruttori di menti e cervelli. il Mulino.

Marjanović, M. J., Scassellati, B., and Williamson, M. M. (1996). Self-taught visually-guided pointing for a humanoid robot. In From Animals to Animats: Proceedings of 1996 Society of Adaptive Behavior, pages $35-44$, Cape Cod, Massachusetts.

Metta, G., Sandini, G., and Konczak, J. (1999). A developmental approach to visually-guided reaching in artificial systems. Neural Networks, 12:1413-1427.

Milner, A. D. and Goodale, M. A. (1995). The visual brain in action. Oxford University Press.

Mussa-Ivaldi, F. A. and Giszter, S. F. (1992). Vector field approximation: a computational paradigm for motor control and learning. $B i$ ological Cybernetics, 67:491-500.

Rizzolatti, G. and Arbib, M. A. (1998). Language within our grasp. Trends in Neurosciences, 21:188-194.

Ungerleider, L. G. and Mishkin, M. (1982). Two cortical visual systems. In Analysis of visual behavior, pages 549-586. MIT Press, Cambridge, Massachusetts.

Williamson, M. (1995). Series elastic actuators. Master's thesis, Massachusetts Institute of Technology, Cambridge, Massachusetts, USA.

Williamson, M. (1998). Neural control of rhythmic arm movements. Neural Networks, 11(78):1379-1394.

Wohlscläger, A. and Bekkering, H. (2002). Is human imitation based on a mirror-neurone system? Some behavioural evidence. Experimental Brain Research, 143:335-341. 